\title{
Medical Image of the Week: Dobhoff Tube Placement with Roux-En-Y Gastric Bypass
}

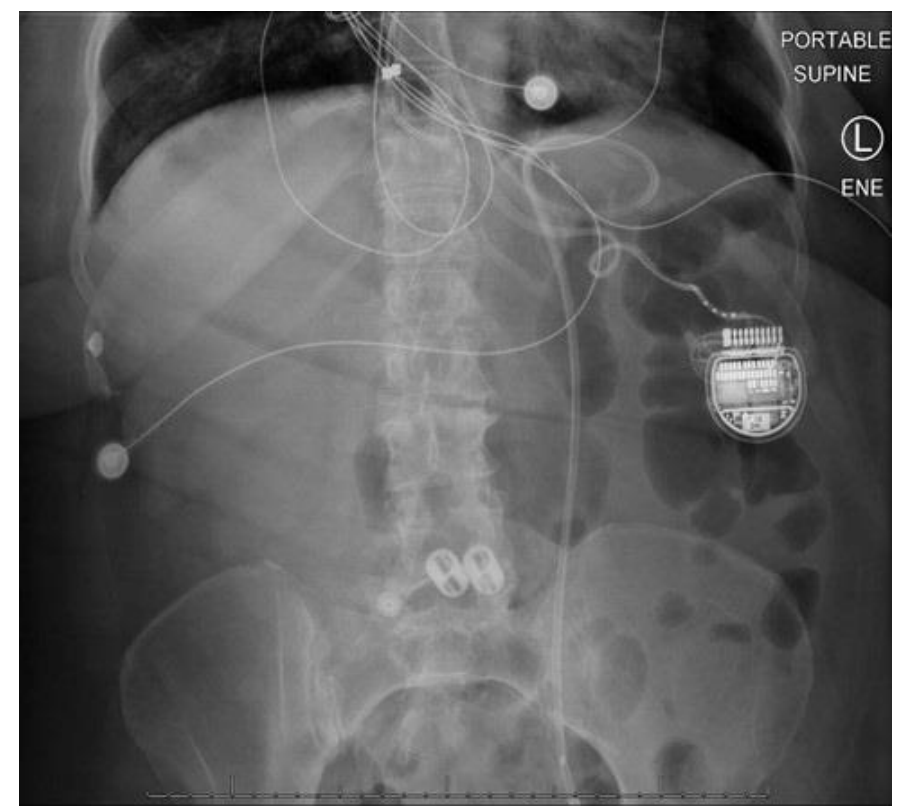

Figure 1. Abdominal X-ray after Dobhoff tube (DHT) placement to confirm accurate positioning. The distal tip of the feeding tube is in a loop of jejunum in patient status post gastrojejunostomy.

\section{Roux-en-Y Gastric Bypass (RNY)}

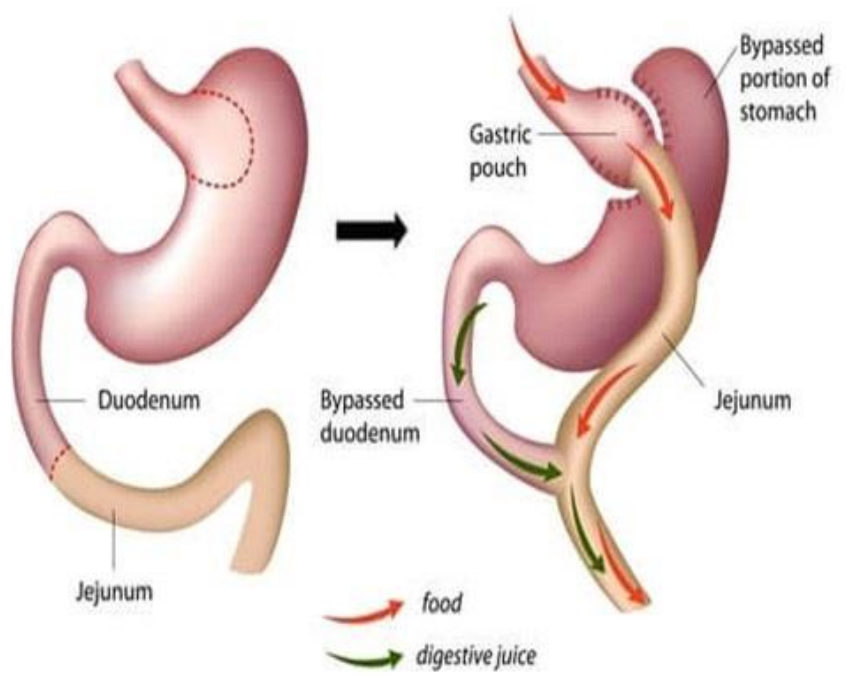

Figure 2. Gastrointestinal anatomy before and after Roux-en-Y gastric bypass procedure. 
Roux-en-Y gastric bypass is one of the most commonly performed bariatric operations in the United States. It involves partitioning a small gastric pouch off the proximal stomach and attaching that pouch directly to the intestine, thereby bypassing the large remainder of the stomach $(1,2)$. Placing a Dobhoff tube (DHT) and confirming its placement can be challenging due to the change in anatomy after the procedure. Here, we have a 65-year-old woman who presented to the hospital with acute encephalopathy and acute hypoxic respiratory failure due to multifocal pneumonia requiring intubation and prolonged ICU stay. A DHT was inserted after intubation for feeding purposes. An abdominal X-ray was obtained to confirm placement of the DHT (Figure 1). Normally the DHT tip should be placed in the $2^{\text {nd }}$ to $3^{\text {rd }}$ portion of the duodenum and would create a C-shaped tracing on the X-ray. However, in our patient who had history of Roux-en-Y, the DHT bypassed the duodenum and went straight down from the stomach to the jejunum as illustrated in Figure 1. It is important to be aware of this change in anatomy in patients who had a Roux-en-Y gastric bypass surgery in order to confirm accurate placement of DHT.

Hasan Ali ${ }^{1}$ MD, Huthayfa Ateeli ${ }^{2}$ MBBS, Bhupinder Natt ${ }^{2}$ MD FACP, and Sachin Chaudhary ${ }^{2} \mathrm{MD}$.

${ }^{1}$ Department of Medicine, Division of Internal Medicine and ${ }^{2}$ Division of Pulmonary, Critical Care, Sleep and Allergy

University of Arizona College of Medicine

Tucson, AZ USA

\section{References}

1. Smoot TM, Xu P, Hilsenrath $P$, Kuppersmith NC, Singh KP. Gastric bypass surgery in the United States, 1998-2002. Am J Public Health. 2006;96(7):1187-9. [CrossRef] [PubMed]

2. Santry HP, Gillen DL, Lauderdale DS. Trends in bariatric surgical procedures. JAMA. 2005;294(15):1909-17. [CrossRef] [PubMed] 